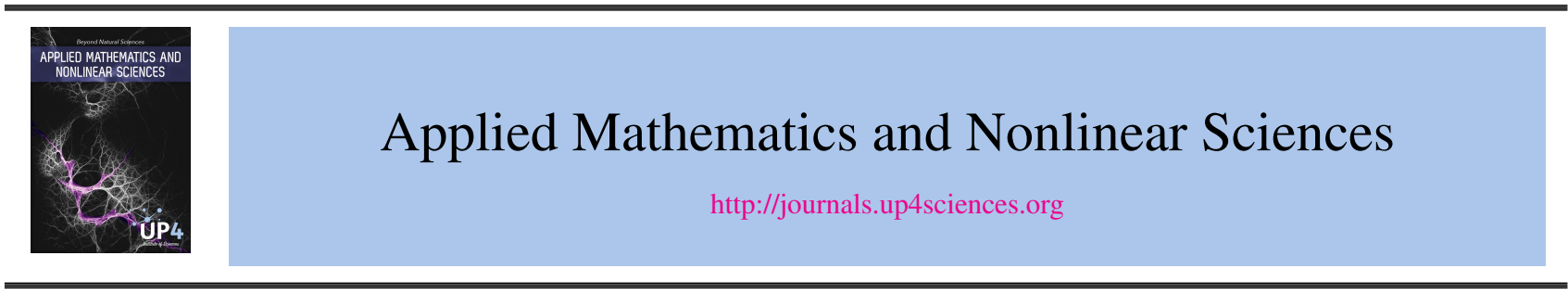

\title{
Friction Torque Behavior as a Function of Actual Contact Angle in Four-point-contact Ball Bearing
}

\section{Chen Long ${ }^{\dagger}$, Xia Xintao, Zheng Haotian, Qiu Ming.}

Henan University of science and technology

Peoples Republic of China

Submission Info

Communicated by Juan L.G. Guirao

Received 17th May 2015

Accepted 1st November 2015

Available online 1st January 2016

\begin{abstract}
The friction is a key factor of rolling bearing performance, especially for those precision transmission fields. All the top rolling bearing manufactures provide their calculating models on friction torque. However, there is great difference between the calculation result and measured value. Two calculation methods on friction torque of four-point-contact ball bearing, considered different factors involving the calculation, are compared and researched for discovering the key influence factor. A specific test is development to verify the theoretical analysis and a precision control method on friction torque with lower cost in production is proposed.
\end{abstract}

Keywords: Friction torque; ball bearing; four-point-contact; contact angle; precision control.

AMS 2010 codes: 00A69.

\section{Symbols}

- $M \quad$ Frictional moment, in newton millimeters;

- $\varphi \quad$ Reduction factor;

- $T$ Variable depending on the bearing type, mean diameter, radial load and axial load;

- $v \quad$ Kinematic viscosity at operating temperature of the oil, in square millimeter per second;

- $n \quad$ Rotational speed, in revolutions per minute;

\section{Corresponding author.}

Email address: *haustchenlong@163.com* 
- $\mu \quad$ Sliding friction coefficient;

- $d \quad$ Diameter on inner ring, in millimeters;

- $V \quad$ Drag loss factor;

- $k \quad$ Related constant;

- $D \quad$ Diameter on outer ring, in millimeters;

- $H$ Oil level, in millimeters;

- $F \quad$ Bearing load, in newtons;

- $Z \quad$ Number of the ball, in pieces;

- $\alpha \quad$ Contact angle, in degree;

- $\omega \quad$ Rotational speed, in $\mathrm{rad} / \mathrm{s}$;

- $R \quad$ Radius, in millimeter;

- $\zeta$ Coefficient of contract material characteristics and geometrical features;

- $\sigma \quad$ Stress, in newtons per square millimeter;

- $\varsigma \quad$ Coefficient of drag loss caused by haulage, turbulence and splash;

- $\varepsilon \quad$ Eccentricity ratio of cage;

- $f \quad$ Raceway groove curvatures factor;

- $G \quad$ Clearance, in micrometer;

- $\beta \quad$ Space angle, in degree;

- $x \quad$ Distance, in millimeter;

- $\Delta \quad$ Variations, in millimeter;

- $E \quad$ Tolerance, in degree / millimeter;

\subsection{Subscripts}

- $r r \quad$ Refers to rolling;

- $s l \quad$ Refers to sliding;

- seal Refers to seals;

- drag Refers to drag loss;

- ish Refers to inlet shear heating;

- rs Refers to kinematic replenishment;

- $p w \quad$ Refers to pitch circle;

- $w \quad$ Refers to the ball; 
- $r \quad$ Refers to radial direction;

- $a \quad$ Refers to axial direction;

- $b l \quad$ Refers to additive package in the lubricant;

- EHL Refers to full-film conditions;

- hys Refers to elastic hysteresis;

- col Refers to cage;

- $i \quad$ Refers to inner ring;

- $o \quad$ Refers to outer ring;

- col-w Refers to cage pocket and balls;

- col-i Refers to inner ring and balls;

- col-o Refers to outer ring and balls;

- poc Refers to cage pockets.

\section{Introduction}

Four-point-contact ball bearings are radial single row angular contact ball bearings with raceways that are designed to support axial loads in both directions. These bearings take up considerably less axial space than double row bearings. The structures of four-point-contact ball bearing are different considering the size of the bearing. For small-size four-point-contact bearing, the inner ring /outer ring is split. This enables a large number of balls to be incorporated in the bearing, giving the bearing its high load carrying capacity. Bearing with split outer rings is defined as QJF000 series and which with split inner rings is defined as QJ000 series in relative standards. They are described in Fig.1 (a) and Fig.1 (b) respectively. For large-size four-point-contact bearings (typically slewing ring bearings, as can be seen in Fig.1 (c)), they have one inner ring and one outer ring. A special filling hole is designed for easily assembling.

The friction in a rolling bearing determines the amount of heat generated by the bearing. The amount of friction depends on the loads and several other factors, including bearing type and size, operating speed, properties and quantity of the lubricant, etc. The friction is a key factor of rolling bearing performance, especially for those precision transmission fields. Snare [1] and [2] presented theoretical investigations of rolling resistance in loaded/unloaded roller/ball bearings, respectively. Top rolling bearing manufactures all provide their calculating model of the frictional moment, as can be found in [3], [4] and [5]. However, the calculating results according to the models are different widely from the actual testing results. Yokoyama et al. [6] evaluated the friction torque of the bearings. Kalin et al. [7] researched friction torque variation of ball bearings owing to different lubrication types and the different inner structures of the bearings. Houerpt [8] compared analytical, numerical and experimental results of ball bearing and tapered roller rearing torque. Wikstrom et al. [9] and Harris et al. [10] analyzed friction torque of bearings by different lubrication types (grease/oil). Joshi et al. [11] and Balamurugan et al. [12] investigated friction torque of bearings running in low/high speeds, respectively. Tiago et al. [13] discussed experimental measuring procedure for friction torque of rolling bearings. Wang [14] analyzed contact angle error in three/four-point-contact bearings and Zupan et al. [15] found the influence of contact angle error on bearing carrying capacity. Chen et al. [16] and [17]discussed the influence of groove shape on clearance in four-point-contact bearings and relative models are established. Florin et al. [18] established a new model which considered the groove shape for calculating friction of ball bearing. Alexandre et al. [19] researched ball 
motion and sliding friction of a four-contact point Bearing.

In this paper, two calculation methods on friction moment are listed and, specific four-point-contact ball bearing is involved in the calculation according to different calculation methods. The main factor that causes different values according to different calculation methods is found and a precision control method on friction torque with lower cost in production is proposed.

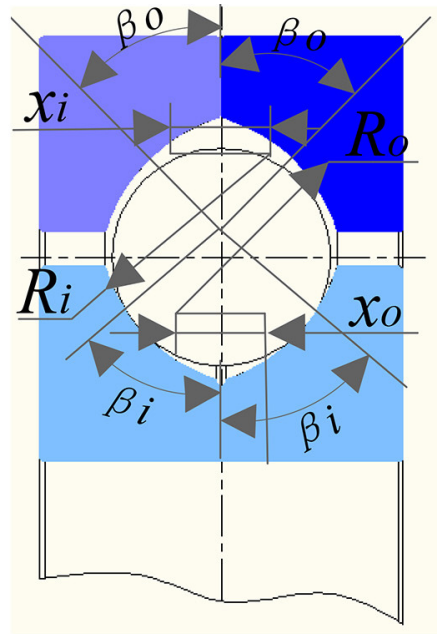

(a) QJ000 Type

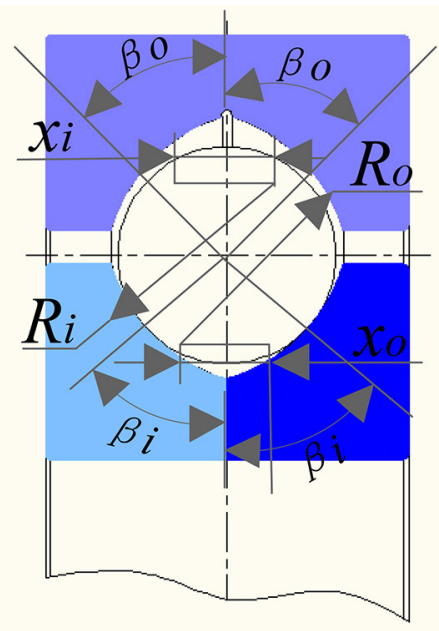

(b) QJF000 Type

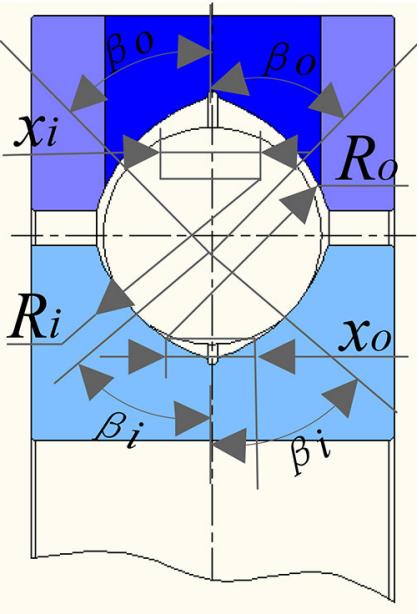

(c) Integrated Type

Fig. 1 Structures of four-point-contact ball bearing

\section{Calculation model of friction torque}

As introduced in the introduction, almost all top rolling bearing manufactures provide their calculating model of the frictional moment. The models from different manufactures are different. However, calculation methods and principles of the models are similar. Hence, only one typical calculation method is chose as calculation tool. In addition, a calculating model different from general calculating models is introduced for discovering the main influence factors of the frictional moment.

\subsection{General modified calculation model}

For four-point-contact ball bearings, a general proposed computational model from bearing manufactures [3], [4] and [5] of friction torque may be approximated by

$$
M=M_{r r}+M_{s l}+M_{\text {seal }}+M_{\text {drag }}
$$

The total frictional moment consists of 4 parts, and they can be expressed as followings respectively

$$
\begin{gathered}
M_{r r}=\varphi_{i s h} \times \varphi_{r s} \times T_{r r} \times(v \times n)^{0.6} \\
M_{s l}=T_{s l} \times m u_{s l} \\
M_{\text {seal }}=0.014 d_{\text {seal }}^{2}+10 \\
M_{\text {drag }}=0.4 v k_{w} D_{p w}^{5} n^{2}+1.9 \times 10^{-9} n^{2} D_{p_{w}}^{3}\left(\frac{n D_{p_{w}}^{2}}{v}\right)^{1.379} D_{p_{w}}^{2}\left\{2 \arccos \left(\frac{0.6 D_{p w}-H}{0.6 D_{p w}}\right)\right. \\
\left.-\sin \left[2 \arccos \left(\frac{0.6 D_{p w}-H}{0.6 D_{p w}}\right)\right]\right\} \frac{3.1(D+d)}{D-d}
\end{gathered}
$$


Variable symbols in (2) can be given as followings respectively

$$
\begin{gathered}
\varphi_{i s h}=\frac{1}{1+1.84 \times 10^{-9}\left(n D_{p w}\right)^{1.28} v^{0.64}} \\
\varphi_{r s}=\frac{1}{e^{6 \times 10^{-8} n v(D+d) \sqrt{\frac{3.1}{D+d}}}} \\
T_{r r}=4.78 \times 10^{-7} D_{p_{w}}^{1.97}\left[F_{r}+1.40 \times 10^{-12} \times D_{p w}{ }^{4} \times n^{2}+2.42 \times F_{a}\right]^{0.54} \\
T_{s l}=1.2 \times 10^{-2} D_{p_{w}}^{0.26}\left[\left(F_{r}+1.40 \times 10^{-12} \times D_{p w}{ }^{4} \times n^{2}\right)^{\frac{4}{3}}+0.9 \times F_{a}^{\frac{4}{3}}\right] \\
\mu_{s l}=\mu_{b l} \frac{1}{e^{6 \times 10^{-8}} n v D p w}+\mu_{E H L}\left(1-\frac{1}{e^{6 \times 10^{-8}} n v D p w}\right)
\end{gathered}
$$

\subsection{Calculation model of considering inner geometric parameters}

Another calculating model of friction torque for four-point-contact ball bearings proposed by Florin T [18] is

$$
M=M_{r r}+M_{s l}+M_{h y s}+M_{c o l}+M_{d r a g}
$$

The total frictional moment consists of 6 parts, and they can be expressed as followings respectively

$$
\begin{gathered}
M_{r r}=\frac{7.8 \pi}{60} D_{w}{ }^{2} Z \sin \alpha\left(\frac{D_{p w}{ }^{2}-D_{w}{ }^{2} \cos ^{2} \alpha}{2 D_{p w} D_{w}}\right) \omega_{i}^{2} \\
M_{s l}=\frac{F D_{p w} Z}{4 D_{w}}\left(1-\frac{D_{w}{ }^{2}}{D_{p w}{ }^{2}} \cos ^{2} \alpha\right)\left(R_{i} \xi_{i}-R_{o} \xi_{o}\right) \mu \\
M_{h y s}=1.25 \times 10^{-4} \times D_{p w} D_{w}^{\frac{2}{3}} \sum_{i=1}^{x} \sigma_{i}^{2} \\
M_{c o l}=M_{c o l-w}+M_{c o l-o}+M_{\text {col }-i} \\
\left\{\begin{array}{l}
M_{\text {drag }}=10^{-7} \times \xi(v n)^{\frac{2}{3}} D_{p w}{ }^{2}(n \geqslant 2000 \mathrm{rpm}) \\
M_{\text {drag }}=16 \times 10^{-6} \times \xi D_{p w}{ }^{2}(n<2000 \mathrm{rpm})
\end{array}\right.
\end{gathered}
$$

Variable symbols in (15) can be given as followings respectively

$$
\begin{gathered}
M_{c o l-w}=\frac{D_{p w}}{4}\left(1-\frac{D_{w}{ }^{2}}{D_{p w}{ }^{2}} \cos ^{2} \alpha\right)\left(\sin \alpha+\tan \frac{D_{w} \sin \alpha}{2 R_{p o c}}\right) \\
M_{c o l-o}=1.38 \times 10^{-4} \times \frac{D_{c o l}}{4} \varepsilon\left(\frac{D_{p w}{ }^{2}-D_{w}{ }^{2} \cos ^{2} \alpha}{2 D_{p w} D_{w}}\right) \\
M_{c o l-i}=1.38 \times 10^{-4} \times \frac{d_{c o l}}{4} \varepsilon\left(\frac{D_{p w}{ }^{2}-D_{w}{ }^{2} \cos ^{2} \alpha}{2 D_{p w} D_{w}}\right)
\end{gathered}
$$


Table 1 Structural parameters of the bearing.

\begin{tabular}{|c|c|c|c|}
\hline Parameter/Unit & Value & Parameter/Unit & Value \\
\hline $\mathrm{O} . \mathrm{R}(D) / \mathrm{mm}$ & 45.225 & $\mathrm{I} . \mathrm{R}(d) / \mathrm{mm}$ & 16.053 \\
\hline Raceway diameter of outer $\operatorname{ring}(D o) / \mathrm{mm}$ & 41.421 & Raceway diameter of inner ring $(d i) / \mathrm{mm}$ & 25.013 \\
\hline $\begin{array}{l}\text { Diameter through the contact point of outer } \\
\operatorname{ring}(D c) / \mathrm{mm}\end{array}$ & 40.635 & $\begin{array}{l}\text { Diameter through the contact point } \\
\text { of inner ring }(d c) / \mathrm{mm}\end{array}$ & 26.626 \\
\hline Width of outer $\operatorname{ring}(\mathrm{Bo}) / \mathrm{mm}$ & 15.5 & Width of inner ring $(B i) / \mathrm{mm}$ & 18.67 \\
\hline Radius of curvature of outer groove $(R o) / \mathrm{mm}$ & 4.2 & Radius of curvature of inner groove $(R i) / \mathrm{mm}$ & 4.2 \\
\hline Diameter of outer ring $\operatorname{rib}(D e) / \mathrm{mm}$ & 38.5 & Diameter of inner $\operatorname{ring} \operatorname{rib}(d i) / \mathrm{mm}$ & 28.5 \\
\hline Contact angle of outer $\operatorname{ring}(\alpha o) /\left(^{\circ}\right)$ & 30 & Contact angle of inner ring $(\alpha i) /\left(^{\circ}\right)$ & 30 \\
\hline Outer diameter of cage $(\mathrm{Dcol}) / \mathrm{mm}$ & 38.2 & Inner diameter of cage $(d c o l) / \mathrm{mm}$ & 31.5 \\
\hline Pocket $\operatorname{radius}(R p o c) / \mathrm{mm}$ & 8 & Diameter of the ball $(D w) / \mathrm{mm}$ & 7.935 \\
\hline Bearing pitch circle diameter $(D p w) / \mathrm{mm}$ & 33.5 & Number of the ball $(Z)$ & 10 \\
\hline
\end{tabular}

\subsection{Comparison of calculating results from different models}

A bearing is chose as example for comparison. The details of structure parameters of the bearing are list as Tab.1. The load of the bearing is set as $10 \mathrm{~N}$ and the rotational speed is set as $100 \mathrm{RPM}$. The lubricant parameters involved in calculation is set as jet fuel No.3. Then friction torque is calculated as $0.0116 \mathrm{~N} \cdot \mathrm{m}$ and 0.0523 $\mathrm{N} \cdot \mathrm{m}$, respectively. Obviously, the calculation results from different calculation models different with each other variously.

By comparison of the two equations, there are four and five components in (1) and (11) respectively. Three factors, Mrr, Msl and Mdrg are same in the two equations. One factor in (1) is not involved in (1), it is the friction moment caused by seals (Mseal). Generally, there is no seal on those four-point-contact ball bearings with high requirement of friction moment. The test bearings have no seal in the paper as well. Hence, Mseal is ignored in the paper. The factors considered in (11) are 2 more than those considered in (1), and they are $M c o l$ and Mhys. Usually, the bearing with cage has better kinetic characteristic. Then it is necessary to consider the friction moments between cage and ring / rolling elements. As for Mhys, Harris [20] discussed the details and developed relative test. The discussion and test results showed that elastic deformation between rolling elements and rings influenced the friction moment of the bearing.

Besides the difference of considered factors, it should be note that the calculation model for calculating same factors in (1) and (11) are different as well, as described in rest equations above-mentioned. The most significant difference of the two models is the different involvement of geometric parameters in calculation. In the first model, only three geometric parameters are involved in, and they are outer diameter $(D)$, inner diameter $(d)$ and pitch circle diameter $(D p w)$, respectively. However, there are much more geometric parameters are involved in the calculation.

\section{Causes of the difference}

The main geometric parameters involved in the 2 nd calculation model are raceway diameters $(R o / R i)$, number of rolling elements $(Z)$, contact angle $(\alpha)$, ball diameter $(D w)$, respectively. There is one special parameter in these parameters, and it is the contact angle $(\alpha)$. The actual contact angle varies with many geometric parameters.

\subsection{Clearance}

Aimed at variety of the actual contact angle, bearing internal clearance is a key parameter which influences the contact angle significantly. Clearance is defined as the total distance through which one bearing ring can 
be moved relative to the other in the radial direction (radial clearance) or in the axial direction (axial clearance).Internal clearance of a bearing is of considerable importance if satisfactory operation is to be obtained. Referring to Fig.2, it shows the impact of initial clearance on contact angle. Fig.2 (a) describes the variation of contact angle owing to radial clearance, and Fig.2 (b) describes the variation owing to axial clearance. The radial clearance can be expressed as

$$
G_{r}=D_{e}-d_{i}-2 D_{w}
$$

The contact angle can be described by radial clearance and geometric parameters as

$$
\alpha=\arccos \left\{1-\frac{G_{r}+\left[\left(2 f_{i}-1\right)\left(1-\cos \beta_{i}\right)+\left[\left(2 f_{o}-1\right)\left(1-\cos \beta_{o}\right)\right] D_{w}\right.}{2\left(f_{i}+f_{o}-1\right) D_{w}}\right\}
$$

In $(21)$

$$
\left\{\begin{array}{l}
\beta_{i}=\arcsin \frac{x_{i}}{\left(f_{i}-0.5\right) D_{w}} \\
\beta_{o}=\arcsin \frac{x_{i}}{\left(f_{o}-0.5\right) D_{w}}
\end{array}\right.
$$

Similarly, the contact angle can be described by axial clearance and geometric parameters as

$$
\alpha=\arccos \frac{G_{a}+2\left(x_{i}+x_{o}\right)}{2\left(f_{i}+f_{o}-1\right) D_{w}}
$$

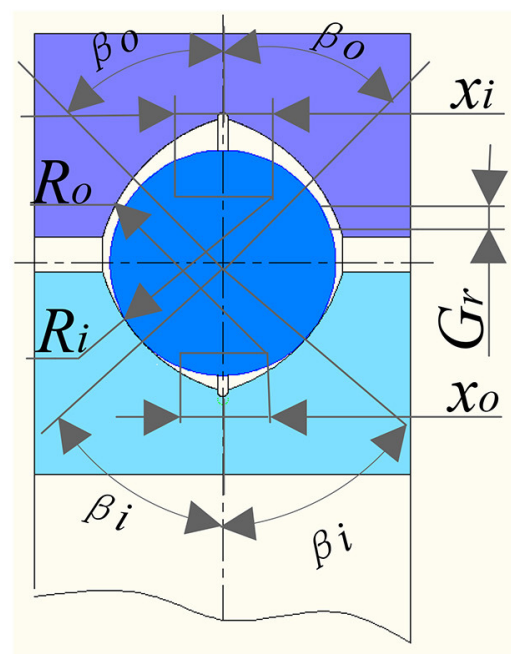

(a) radial clearance

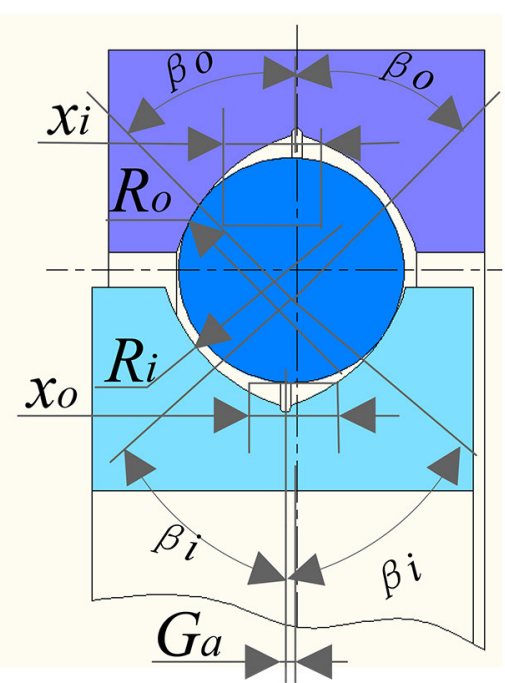

(b) axial clearance

Fig. 2 contact angle variation owing to the clearance.

\subsection{Distance between groove curvature centres}

In regard of radial clearance, the space angle $(\beta o / \beta i)$ is influenced by the distance between groove curvature centers $(x o / x i)$ directly according to (21), and the contact angle $(\alpha)$ is influenced by the space angle according to (21). Correspondingly, the contact angle is influenced by the distance between groove curvature centres directly according to (32) in regard of axial clearance.

In order to describe the variation of space angle briefly, a four-point-contact bearing with zero clearance is chosen as research object, as described in Fig. 3 (a). The groove curvatures of inner ring and outer ring are $R i$ and $R o$, respectively. The distance between groove curvature centres of the inner ring is $x i$ and the distance 
between groove curvature centres of the outer ring is $x o$. When the distances change into $x i$, and $x o^{\prime}$, the space angles turn into $\beta i$, and $\beta o^{\prime}$. In addition, the ball diameter changes from $D w$ into $D w^{\prime}$. Actually, the size of the ball is consistent in the bearing. Then the variation of the ball diameter passes on to the clearance. We can draw out conclusion that the distance between groove curvature centres influences both contact angle and clearance.

\subsection{Groove curvature}

According to 13, the value of groove curvature influences the friction moment caused by sliding directly. In addition, the contact angle is influenced by groove curvature according to 13 and 13), and the value of groove curvature is the other impact factor on friction moment. As can be seen in 1, the groove curvatures of inner ring and outer ring change from $R i$ and $R o$ to $R i$, and $R o^{\prime}$. The space angles turn into $\beta i$, and $\beta o^{\prime}$, and the clearance will be smaller. The reason is the same as analysis in 3.2.

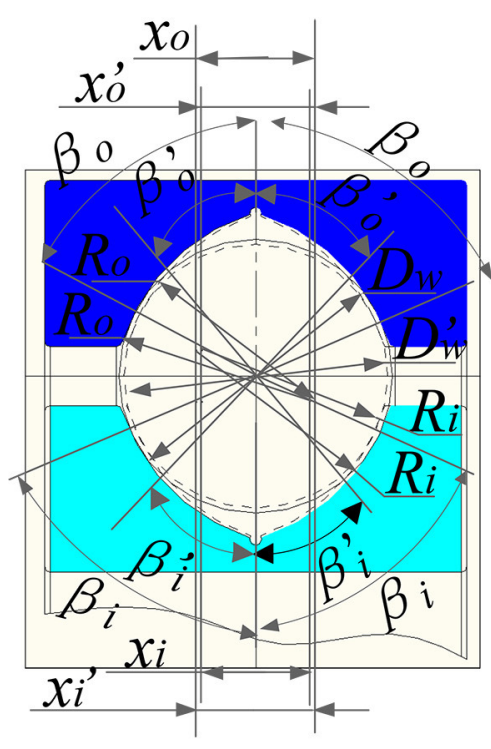

(a) distance

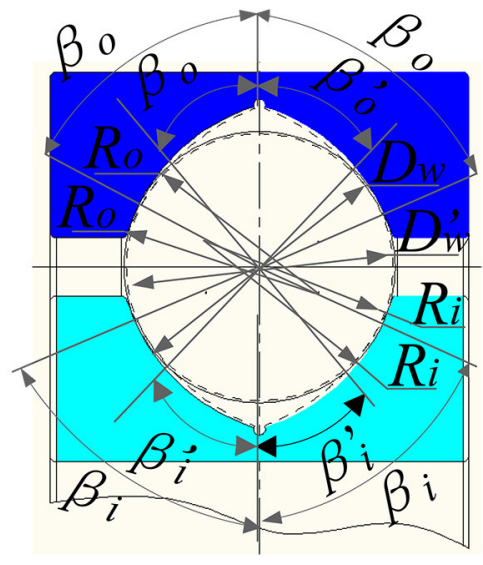

(b) curvature

Fig. 3 contact angle variation owing to groove.

\section{Test}

Then a special test on actual friction torque is developed for further investigation. 10 pieces four-pointcontact bearings are selected to test the friction torques and inner geometric parameters. The parameters are listed in Tab.2.

\subsection{Test program}

The special test developed for verifying the causing factors of friction variations is divided into 4 steps as following. The 1st step is assembling the bearing. Radial clearances of the bearings are checked. The 2nd step is testing the actual friction torque of the bearing. The load on bearings under test is $10 \mathrm{~N}$. It has the inner ring fixed vertically and the outer ring rotated around vertical axis. Rotating speed of the outer ring is 100 RPM in test process. The 3rd step is testing the contact angles of inner rings and out rings by profilometer. It should be noted that both rings have two raceways for four-point-contact bearing. Then there are four measurement results for every bearing. In addition, there are various kinds of profilometers in the market now. However, only a few 
profilometers possess the function of calculating the contact angle. The basic principle of the profilometer is that a circle having same diameter of the balls is inscribed with the two curves having been drawn by the profilometer, and then the contact angle can be calculated out. 1600D profilometer from Tokyo Seimitsu is applied in the test. The 4th step is testing the contact angles of inner rings and out rings by horizontal metroscope. Mahr828PC from Mahr $\mathrm{GmbH}$ is applied in the test. Measuring error of the equipment is smaller than $0.04+\mathrm{L} / 2000$ and measurement uncertainty of the equipment is $0.15+\mathrm{L} / 1000$. It should be noted that measurement head of the equipment was a feeler pin before. In order to test the test the distance between contact points, a ball with diameter of $7.9375 \mathrm{~mm}$ is welded on the end of measuring rod for convenience of measurement. Spherical part of the steel ball is $-2 \mu m$.

\subsection{Test Results}

Test results and the calculated results according to tested contact angel of friction torque can be seen in Tab. 2 as well. The contact angles tested by profilometer are list as two groups' data. They are contact angles of upper groove and lower groove, respectively. The contact angles tested by metroscope are list as one group.

1. The test results indicate that theoretical models of friction torque are imperfect. As introduced in 2.2, friction torques are calculated by different theoretical models as $0.0116 \mathrm{~N} \cdot \mathrm{m}$ and $0.0523 \mathrm{~N} \cdot \mathrm{m}$, respectively. However, the mean value of measured data is $0.1223 \mathrm{~N} \cdot \mathrm{m}$. The mean value of measured data is nearly 10.54 times of calculated value through 1 st theoretical model, and it is nearly 2.34 times of calculated value through 2 nd theoretical model.

2. As for the test results of contact angle from different test methods, the mean values tested by profilometer is greater than the values by metroscope. The cause of the different measurement values is the difference of principle between the two measurement methods. The theoretical circle by profilometer has two contact points with the inner/outer ring at any position. However, the steel ball welded on the end of measuring rod has only one contact point owing to the horizontal position is defined.

3. The mean value of calculated results with tested contact angel is $0.10 \mathrm{~N} \cdot \mathrm{m}$. It is much higher than calculated results from general model, and it is very close the test value. This indicates that actual contact angel is the key factor which influences the friction torque.

4. The tested contact angles of NO.1, 2 and 5 is nearly. As a result, the test results of friction torque are near. This is the other proof that the actual contact angel is the key factor which influences the friction torque.

5. The calculated results of friction torque are still smaller than the test values. The main reason maybe the roundness error.

\section{Control method}

According to above analysis, we found the significant importance of actual contact angel. As a matter of fact, the variation of actual contact angel is determined by accuracy of rings and balls. Hence, aim at different requirements of friction torque, bearings with different accuracy will be chosen. However, bearings with higher class imply much higher cost. If the relations between friction torque and dimensions can be found and the impact density can be quantized, these dimensions will be controlled in production purposefully. And, the production cost will be sharply reduced. In different applications, clearance in different directions (radial / axial) is required. Then the different requirements are discussed as following. For radial clearance, differentiating (21) with respect to radial clearance, distance between groove curvature centers and the groove curvatures, we have (24) 
Table 2 Structural parameters of the bearing.

\begin{tabular}{|c|c|c|c|c|c|c|c|c|c|c|c|}
\hline \multirow[t]{2}{*}{ Serial } & \multicolumn{3}{|c|}{$\begin{array}{l}\text { Space Angle of grooves } \\
\text { on I.R } \\
\left({ }^{\circ}\right)\end{array}$} & \multicolumn{3}{|c|}{$\begin{array}{l}\text { Space Angle of grooves } \\
\text { on O.R } \\
\left({ }^{\circ}\right)\end{array}$} & \multicolumn{2}{|c|}{$\begin{array}{l}\text { Contact } \\
\text { Angle } \\
\left({ }^{\circ}\right)\end{array}$} & \multirow[t]{2}{*}{$\begin{array}{l}\text { Clea- } \\
\text {-rance } \\
\mu \cdot \mathrm{m}\end{array}$} & \multicolumn{2}{|c|}{$\begin{array}{l}\text { Friction } \\
\text { Torque } \\
\mathrm{N} \cdot \mathrm{m}\end{array}$} \\
\hline & Upper & Lower & Mean & Upper & Lower & Mean & O.R & I.R & & test & calculate \\
\hline 1 & 27.42 & 27.54 & 27.48 & 29.23 & 27.07 & 28.15 & 27.45 & 28.85 & 10 & 0.105 & 0.084 \\
\hline 2 & 30.45 & 28.12 & 29.28 & 29.50 & 26.97 & 28.23 & 28.50 & 28.35 & 14 & 0.098 & 0.072 \\
\hline 3 & 30.54 & 28.36 & 29.45 & 27.77 & 29.67 & 28.71 & 29.65 & 29.70 & 10 & 0.140 & 0.131 \\
\hline 4 & 30.64 & 29.08 & 29.86 & 29.29 & 27.85 & 28.56 & 29.85 & 29.00 & 11 & 0.126 & 0.102 \\
\hline 5 & 31.38 & 28.77 & 30.07 & 28.29 & 29.00 & 28.64 & 29.25 & 28.35 & 11 & 0.109 & 0.096 \\
\hline 6 & 30.99 & 28.87 & 29.93 & 28.40 & 25.73 & 27.06 & 29.55 & 26.15 & 10 & 0.130 & 0.109 \\
\hline 7 & 30.35 & 28.72 & 29.54 & 28.28 & 28.06 & 28.17 & 29.15 & 28.55 & 12 & 0.137 & 0.114 \\
\hline 8 & 30.40 & 28.57 & 29.49 & 27.28 & 28.29 & 27.78 & 29.05 & 28.50 & 11 & 0.133 & 0.107 \\
\hline 9 & 30.59 & 28.79 & 29.69 & 28.80 & 27.82 & 28.31 & 29.20 & 28.20 & 8 & 0.119 & 0.098 \\
\hline 10 & 30.77 & 28.78 & 29.77 & 29.90 & 27.18 & 28.54 & 29.25 & 27.50 & 12 & 0.126 & 0.101 \\
\hline
\end{tabular}

$$
\left\{\begin{array}{l}
\frac{\partial \alpha}{\partial x_{i}}=\frac{\tan \beta_{i}}{\left(R_{o}+R_{i}-D_{w}\right) \sin \alpha} \\
\frac{\partial \alpha}{\partial x_{o}}=\frac{\tan \beta_{o}}{\left(R_{o}+R_{i}-D_{w}\right) \sin \alpha} \\
\frac{\partial \alpha}{\partial G_{r}}=\frac{1}{2\left(R_{o}+R_{i}-D_{w}\right) \sin \alpha} \\
\frac{\partial \alpha}{\partial R_{i}}=\frac{\cos \alpha-\cos \beta_{i}-\tan \beta_{i} \sin \beta_{i}}{\left(R_{o}+R_{i}-D_{w}\right) \sin \alpha} \\
\frac{\partial \alpha}{\partial R_{o}}=\frac{\cos \alpha-\cos \beta_{o}-\tan \beta_{o} \sin \beta_{o}}{\left(R_{o}+R_{i}-D_{w}\right) \sin \alpha} \\
\frac{\partial \alpha}{\partial D_{w}}=\frac{\tan \alpha}{R_{o}+R_{i}-D_{w}}
\end{array}\right.
$$

The variation of the contact angle can be expressed as

$$
\Delta \alpha=\frac{\partial \alpha}{\partial G_{r}} \Delta G_{r}+\frac{\partial \alpha}{\partial x_{i}} \Delta x_{i}+\frac{\partial \alpha}{\partial x_{o}} \Delta x_{o}+\frac{\partial \alpha}{\partial R_{o}} \Delta R_{o}+\frac{\partial \alpha}{\partial R_{i}} \Delta R_{i}++\frac{\partial \alpha}{\partial D_{w}} \Delta D_{w}
$$

Then the design tolerance can be described as

$$
E_{\alpha r}=\left|\frac{\partial \alpha}{\partial G_{r}}\right| E_{G r}+\left|\frac{\partial \alpha}{\partial x_{i}} \Delta x_{i}\right| E_{x i}+\left|\frac{\partial \alpha}{\partial x_{0}} \Delta x_{o}\right| E_{x o}+\left|\frac{\partial \alpha}{\partial R_{i}} \Delta R_{i}\right| E_{R i}+\left|\frac{\partial \alpha}{\partial R_{o}} \Delta R_{o}\right| E_{R o}+\left|\frac{\partial \alpha}{\partial D_{w}} \Delta D_{w}\right| E_{D w}
$$

In addition, the variation of space angels $(\alpha \mathrm{i}$ and $\alpha \mathrm{o})$ should be control as well. Differentiating (21) with respect to relative variables, we have (27).

The variation of the space angle is

$$
\left\{\begin{array}{l}
\frac{\partial \beta_{i}}{\partial x_{i}}=\frac{1}{\left(R_{i}-\frac{D_{w}}{2}\right) \cos \beta_{i}} \\
\frac{\partial \beta_{i}}{\partial R_{i}}=-\frac{\operatorname{tg} \beta_{i}}{R-\frac{D_{w}}{2}} \\
\frac{\partial \beta_{i}}{\partial D_{w}}=-\frac{\operatorname{tg} \beta_{i}}{2\left(R-\frac{D_{w}}{2}\right)} \\
\frac{\partial \beta_{o}}{\partial x_{o}}=\frac{1}{\left(R_{o}-\frac{D_{w}}{2}\right) \cos \beta_{o}} \\
\frac{\partial \beta_{o}}{\partial R_{o}}=-\frac{\operatorname{tg} \beta_{o}}{R_{o}-\frac{D_{w}}{w_{w}}} \\
\frac{\partial \beta_{o}}{\partial D_{w}}=-\frac{\operatorname{tg} \beta_{o}}{2\left(R_{o}-\frac{D_{w}}{2}\right)}
\end{array}\right.
$$

$$
\left\{\begin{array}{l}
\Delta \beta_{i}=\frac{\partial \beta_{i}}{\partial x_{i}} \Delta x_{i}+\frac{\partial \beta_{i}}{\partial R_{i}} \Delta R_{i}+\frac{\partial \beta_{i}}{\partial D_{w}} \Delta D_{w} \\
\Delta \beta_{o}=\frac{\partial \beta_{o}}{\partial x_{o}} \Delta x_{o}+\frac{\partial \beta_{o}}{\partial R_{o}} \Delta R_{o}+\frac{\partial \beta_{o}}{\partial D_{w}} \Delta D_{w}
\end{array}\right.
$$


And the design tolerance should be

$$
\left\{\begin{array}{l}
E_{\beta i}=\left|\frac{\partial \beta_{i}}{\partial x_{i}}\right| E_{x i}+\left|\frac{\partial \beta_{i}}{\partial R_{i}}\right| E_{R i}+\left|\frac{\partial \beta_{i}}{\partial D_{w}}\right| E_{D w} \\
E_{\beta o}=\left|\frac{\partial \beta_{o}}{\partial x_{o}}\right| E_{x o}+\left|\frac{\partial \beta_{o}}{\partial R_{o}}\right| E_{R o}+\left|\frac{\partial \beta_{o}}{\partial D_{w}}\right| E_{D w}
\end{array}\right.
$$

For axial clearance, differentiating (32) with respect to axial clearance, distance between groove curvature centers and the groove curvatures, integration of equation can be obtained.

$$
\left\{\begin{array}{l}
\frac{\partial \alpha}{\partial x_{i}}=\frac{\tan \beta_{i}}{\left(R_{o}+R_{i}-D_{w}\right) \sin \alpha} \\
\frac{\partial \alpha}{\partial x_{o}}=\frac{\tan \beta_{o}}{\left(R_{o}+R_{i}-D_{w}\right) \sin \alpha} \\
\frac{\partial \alpha}{\partial G_{r}}=\frac{1}{2\left(R_{o}+R_{i}-D_{w}\right) \sin \alpha} \\
\frac{\partial \alpha}{\partial R_{i}}=\frac{\cos \alpha-\cos \beta_{i}-\tan \beta_{i} \sin \beta_{i}}{\left(R_{o}+R_{i}-D_{w}\right) \sin \alpha} \\
\frac{\partial \alpha}{\partial R_{o}}=\frac{\cos \alpha-\cos \beta_{o}-\tan \beta_{o} \sin \beta_{o}}{\left(R_{o}+R_{i}-D_{w}\right) \sin \alpha} \\
\frac{\partial \alpha}{\partial D_{w}}=\frac{\tan \alpha}{R_{o}+R_{i}-D_{w}}
\end{array}\right.
$$

The variation of the contact angle can be written as follows

$$
\Delta \alpha=\frac{\partial \alpha}{\partial G_{a}} \Delta G_{r}+\frac{\partial \alpha}{\partial x_{i}} \Delta x_{i}+\frac{\partial \alpha}{\partial x_{o}} \Delta x_{o}+\frac{\partial \alpha}{\partial R_{o}} \Delta R_{o}+\frac{\partial \alpha}{\partial R_{i}} \Delta R_{i}++\frac{\partial \alpha}{\partial D_{w}} \Delta D_{w}
$$

Then the design tolerance can be given as

$$
E_{\alpha a}=\left|\frac{\partial \alpha}{\partial G_{a}}\right| E_{G a}+\left|\frac{\partial \alpha}{\partial x_{i}} \Delta x_{i}\right| E_{x i}+\left|\frac{\partial \alpha}{\partial x_{0}} \Delta x_{o}\right| E_{x o}+\left|\frac{\partial \alpha}{\partial R_{i}} \Delta R_{i}\right| E_{R i}+\left|\frac{\partial \alpha}{\partial R_{o}} \Delta R_{o}\right| E_{R o}+\left|\frac{\partial \alpha}{\partial D_{w}} \Delta D_{w}\right| E_{D w}
$$

\section{Conclusions}

The theoretical analysis and compassion tests can be summarized to give the following conclusions 1. Comparison of the calculated results on friction torque according to two different calculation methods shows that the friction torque is deeply influenced by actual inner geometric parameters.

2. The key factor of which influence the friction torque mostly is confirmed as the actual contact angle. The actual contact angle is influenced by various factors including radial clearance, raceway groove curvatures and groove profile.

3. The calculation results according to actual contact angel by specific test are closer to the test results. Then actual contact angel can be determined as the key factor which influences the friction torque.

4. A precision control method on friction torque with lower cost is proposed. Then the requirements of friction torque can be controlled in the process of design / production circle.

Two questions are not solved completely in this paper, and the subsequent research should be developed for more complete conclusions.

1. Generally, bearing clearance is divided into initial clearance, mounting clearance and operational clearance, respectively. This means that clearance is a variable according to different application stage. The clearances involved in the calculation are initial clearance. The operational clearance in actual application is the best choice for calculation.

2. Roundness error is supposed to be another impact factor on friction torque. But the conclusion needs the specific test to verify. 


\section{Acknowledgements}

The project is supported by National Natural Science Foundation of China (Grant No.51475144) and the Foundation of Innovation and Research Team of Science and Technology in Universities in Henan Province (Grant No. 13IRTSTHN025).

\section{References}

[1] Snare B., (1968) Rolling Resistance in Lighty Loaded Bearings, The Ball Bearing Journal,152, 3-8.

[2] Snare B., (1968) Rolling Resistance in Loaded Roller Bearings, The Ball Bearing Journal, 153, 19-24.

[3] SKF (2014), Rolling Bearing. PUB/P1 10000 EN, Sweden: SKF 2014.

[4] NSK (2013), Rolling Bearing. CAT. No.CH1103A 2013 E-11, Japan: NSK 2013.

[5] Schaeffler KG (2008), Rolling Bearing. Publication - FAG Australia Pty Ltd, Australia: FAG 2005.

[6] Yokoyama K., Tohyama A., Suzuki T., (1996), Evaluation of Friction Torque of Rolling Bearing , Journal of the Japan Society for Precision Engineering, Vol 62(2), 210-214.

[7] Kalin M, Vizintin J., (1997), Influence of the Lubrication Type and the Structure of Friction Torque on Total Friction in Ball Bearings. Journal of mechanical engineering, Vol 43(5), 231-238.

[8] Houerpt L., (2002), Ball Bearing and Tapered Roller Bearing Torque: Analytical, Numerical and Experimental Results. Tribology Transactions, Vol 45(3), 156-163. doi 10.1080/10402000208982559

[9] Wikstrom V, Hoglund E., (1996), Starting and Steady-state Friction Torque of Grease Lubricated Rolling Element Beatings at Low Temperatures - Part I: a Parameter Study. Tribology Transactions, 39(3), 517-526. doi $10.1080 / 10402009608983562$

[10] Harris T. A., Barsby R. M., Kotzalas M. N., (2011), A Method to Calculate Frictional Effects in Oil-lubricated Ball Bearings. Tribology Transactions, 44(4), pp704-708. doi 10.1080/10402000108982514

[11] Joshi, A., 1, Kachhia B. and 1;2, Kikkari, H., et al (2015), Running Torque of Slow Speed Two-Point and Four-Point Contact Bearings. Lubricants 3, 181-196. doi 10.3390/lubricants3020181

[12] Balamurugan.N, C.Bhagyanathan ., (2014), Analysis and Investigation on Thermal Behaviors of Ball Bearing in High Speed Spindle. International Journal of Innovative Science and Modern Engineering, 2(4), 16-20.

[13] Tiago C., Beatriz G., Armando C. and Jorge S., (2010), Experimental measuring procedure for the friction torque in rolling bearings, Lubrication Science, 22(4), 133-147. doi 10.1002/1s.115

[14] Wang H., (1998), Design error analysis of contact angle in three/four-point-contact bearings. Bearing, 7, 2-7 (in Chinese).

[15] Zupan S, Prebil I (2001). Carrying Angel and Carrying Capacity of a large single row ball bearing as a function of geometry parameters of the rolling contact and the supporting structure stiffness. Mechanism and Machine Theory, 36(10), 1087-1103. doi 10.1016/S0094-114X(01)00044-1

[16] Chen L., Jiang H. W., Wang L. F. et, al., (2012), Characteristic Analysis on Relationship between Groove Profile and Clearance in Four-Point-Contact Ball Slewing Bearings. Bearing, 6, 32-36,62 (in Chinese).

[17] Chen L., Li Z. G., Qiu M., Xia X. T., (2014), Influence of groove shape on clearance in four-point-contact slewing bearing. Journal of the Brazilian Society of Mechanical Sciences and Engineering, 36(3), 461-467. doi 10.1007/s40430013-0118-7

[18] Florin T, Barbu Dr (2002)., Computing Race Ways Shape of Ball Bearing. The annals of university, 3, 99-103.

[19] Alexandre L. Danie1N., (2007), Ball Motion and Sliding Friction on a Four-contact Point Ball Bearing. Journal of Tribology, 129(4), 801-808. doi 10.1115/1.2768079

[20] Harris T.A., Kotzalas M. N., (2006), Rolling Bearing Analysis, fifth edition. Taylor and Francis Group, LLC.

OUP4 Sciences. All rights reserved. 